\title{
ON THE BIRATIONAL GEOMETRY OF MODULI SPACES OF POINTED CURVES
}

\author{
E. Ballico, G. Casnati, and C. Fontanari
}

\begin{abstract}
We prove that the moduli space $\mathcal{M}_{g, n}$ of smooth curves of genus $g$ with $n$ marked points is rational for $g=6$ and $1 \leq n \leq 8$, and it is unirational for $g=8$ and $1 \leq n \leq 11$, $g=10$ and $1 \leq n \leq 3, g=12$ and $n=1$.
\end{abstract}

\section{INTRODUCTION AND NOTATION}

Let $\mathcal{M}_{g, n}$ be the (coarse) moduli space of smooth curves of genus $g$ with $n$ marked points defined over the field $\mathbb{C}$ of complex numbers and $\overline{\mathcal{M}}_{g, n}$ its Deligne-Mumford compactification. Even if one is mainly interested in the geometry of $\overline{\mathcal{M}}_{g}$ (i.e. the case $n=0$ ), it is worthwhile to consider pointed moduli spaces as well. Indeed, the boundary components of $\overline{\mathcal{M}}_{g}$ are images via natural gluing morphisms of pointed moduli spaces of lower genus.

As an example of this philosophy applied in the specific context of birational geometry, we just mention the nice contribution $[\mathrm{B}-\mathrm{V}]$ : in order to prove that $\overline{\mathcal{M}}_{15}$ is rationally connected, a key ingredient (see Theorem 4.7) is provided by the fact that $\overline{\mathcal{M}}_{14,2}$ is unirational.

Several results about the Kodaira dimension $\kappa\left(\overline{\mathcal{M}}_{g, n}\right)$ of $\overline{\mathcal{M}}_{g, n}$ are already known thanks to the papers $[\mathrm{Be}],[\mathrm{B}-\mathrm{F}],[\mathrm{B}-\mathrm{V}],[\mathrm{Fa}]$ and $[\mathrm{Lg}]$. In particular, in the above contributions it is proved that $\overline{\mathcal{M}}_{g, n}$ is unirational for $1 \leq n \leq \sigma(g)$ and $\kappa\left(\overline{\mathcal{M}}_{g, n}\right) \geq 0$ for $n \geq \tau(g)$ where the values of $\sigma$ and $\tau$ are as follows

\begin{tabular}{|c|c|c|c|c|c|c|c|c|c|c|c|c|c|c|c|c|c|c|c|c|c|}
\hline$g$ & 1 & 2 & 3 & 4 & 5 & 6 & 7 & 8 & 9 & 10 & 11 & 12 & 13 & 14 & 15 & 16 & 17 & 18 & 19 & 20 & 21 \\
\hline$\sigma(g)$ & 10 & 11 & 14 & 15 & 12 & 15 & 11 & 5 & 8 & & 10 & & & 2 & & & & & & & \\
\hline$\tau(g)$ & 11 & & & 16 & 15 & 16 & 14 & 14 & 13 & 11 & 11 & 13 & 11 & 10 & 10 & 9 & 9 & 9 & 7 & 6 & 4 \\
\hline
\end{tabular}

Notice that only for few values of $g$ (namely $g=1,4,6$ and 11) such results are tight.

For small $g$ even the rationality of $\mathcal{M}_{g, n}$ can be proved. More precisely $\mathcal{M}_{g, n}$ is rational for $g=1$ and $1 \leq n \leq 10$ (see [Be]), $g=2$ and $1 \leq n \leq 12, g=3$ and $1 \leq n \leq 14, g=4$ and $1 \leq n \leq 15, g=5$ and $1 \leq n \leq 12$ (see $[\mathrm{C}-\mathrm{F}]$ ).

In the present paper our first main theorem concerns the rationality of $\mathcal{M}_{6, n}$.

2000 Mathematics Subject Classification. 14H10, 14H45, 14E08, 14L30.

Key words and phrases. Pointed curve, Moduli space, Rationality, Unirationality.

This research was partially supported by MIUR and GNSAGA of INdAM (Italy). 
Theorem A. The moduli space $\mathcal{M}_{6, n}$ is rational for $1 \leq n \leq 8$.

Our key idea is to mix the techniques in $[\mathrm{S}-\mathrm{B}]$, where the rationality of $\mathcal{M}_{6}$ was originally proven, with those introduced in $[\mathrm{C}-\mathrm{F}]$.

In higher genera we are able to improve the result of [Lg] in some cases, according to our second main theorem.

Theorem B. The moduli space $\mathcal{M}_{g, n}$ is unirational for $g=8$ and $1 \leq n \leq 8, g=10$ and $1 \leq n \leq 3, g=12$ and $n=1$.

Our methods are miscellaneous in nature and involve different ad hoc constructions for each genus. In particular, for $g=8$ we embed the general curve on a quadric surface via two distinct $g_{5}^{1}$; for $g=10$ we apply the classical construction of Beniamino Segre revisited in $[\mathrm{A}-\mathrm{C}]$; finally, for $g=12$ we follow the lines of the unirationality arguments presented in $[\mathrm{Ve}]$.

We are grateful to Igor Dolgachev, who shared with us his deep insight into rationality questions in a stimulating and inspiring conversation held in Turin during the School (and Workshop) on Cremona transformations (September 2005).

Notation. We work over the field $\mathbb{C}$ of complex numbers. Projective $r$-space will be denoted by $\mathbb{P}_{\mathbb{C}}^{r}$.

If $X$ is a projective scheme, then a divisor $D$ on $X$ is always intended to be a Cartier divisor and $|D|$ denotes the complete linear system of divisors linearly equivalent to $D$.

A curve $C$ is a projective scheme of dimension 1. If $D$ is a divisor on $C$ then $\varphi_{|D|}$ denotes the map induced on $C$ by $|D|$. If $C, D \subset \mathbb{P}_{\mathbb{C}}^{2}$ are distinct plane curves, then $C \cdot D$ denotes their intersection cycle.

Let $\mathcal{M}_{g, n}$ be the coarse moduli space of smooth, projective $n$-pointed curves (briefly $n$-pointed curves in what follows) of genus $g$. A point of $\mathcal{M}_{g, n}$ is then the isomorphism class of a $(n+1)$-tuple $\left(C, p_{1}, \ldots, p_{n}\right)$, where $C$ is a smooth and connected curve of genus $g$ and $p_{1}, \ldots, p_{n} \in C$. As usual, we set $\mathcal{M}_{g}:=\mathcal{M}_{g, 0}$. A general curve of genus $g$ is a curve corresponding to the general point of the irreducible scheme $\mathcal{M}_{g}$.

We denote isomorphisms by $\cong$ and birational equivalences by $\approx$. For other definitions, results and notation we always refer to $[\mathrm{Ha}]$.

\section{The rationality of $\mathcal{M}_{6,1}$}

Let $\left(C, p_{1}\right) \in \mathcal{M}_{6,1}$ be a general point: in particular $C$ is a general curve of genus 6 . Following [SB], we notice that the canonical system $|K|$ embeds $C$ in $\mathbb{P}_{\mathbb{C}}^{5}$ as a curve $C_{K}$ of degree 10 as the intersection of a unique del Pezzo surface $S_{5} \subseteq \mathbb{P}_{\mathbb{C}}^{5}$ of degree 5 with a quadric. We recall that such an $S$ is the blow up of $\mathbb{P}_{\mathbb{C}}^{2}$ at four general points embedded in $\mathbb{P}_{\mathbb{C}}^{5}$ via the linear system $\Sigma$ of cubic divisors in $\mathbb{P}_{\mathbb{C}}^{2}$ through the four points.

Since each two sets of four general points in $\mathbb{P}_{\mathbb{C}}^{2}$ are projectively equivalent, we can always assume that such four points are the fundamental points $E_{1}, E_{2}, E_{3}, E_{4}$ and let $s: S \rightarrow \mathbb{P}_{\mathbb{C}}^{2}$ be the corresponding blow up morphism. In particular, we can fix once and for all the surface $S$ and such a representation of $S$ allows us to consider insted of $C_{K}$ its plane model $\bar{C}:=s\left(C_{K}\right) \subseteq \mathbb{P}_{\mathbb{C}}^{2}$ as plane sextic curve with nodes at the points $E_{1}, E_{2}, E_{3}, E_{4}$.

We finally recall that $\operatorname{Aut}(S) \cong \mathfrak{S}_{5}$ : in the plane representation of $S$, it coincides with the rational representation of $\mathfrak{S}_{5}$ as the group generated by the projectivities fixing the 
set $E:=\left\{E_{1}, E_{2}, E_{3}, E_{4}\right\}$ (which is isomorphic to $\mathfrak{S}_{4}$ ) and by the standard quadratic transformation

$$
\mu\left(x_{1}, x_{2}, x_{3}\right)=\left(x_{2} x_{3}, x_{1} x_{3}, x_{1} x_{3}\right) .
$$

Remark 1.1. Let $\Psi: C \stackrel{\sim}{\longrightarrow} C^{\prime}$ be an isomorphism of abstract 1 -pointed curves of genus 6 mapping the point $p_{1} \in C$ to $p_{1}^{\prime} \in C^{\prime}$. It carries the canonical system $K$ of $C$ onto the one $K^{\prime}$ of $C^{\prime}$. Thus $\Psi$ induces a projectivity of the canonical space mapping $C_{K}$ to $C_{K^{\prime}}^{\prime}$ : obviously such a projectivity must map the unique del Pezzo surface through $C$, which is $S$, to the one through $C^{\prime}$, which is again $S$.

Thus $\Psi$ induces via $s$ an element $\sigma \in \mathfrak{S}_{5}$ mapping $\bar{C}$ to $\overline{C^{\prime}}:=s\left(C_{K^{\prime}}^{\prime}\right)$ and $A_{1}:=s\left(p_{1}\right)$ to $A_{1}^{\prime}:=s\left(p_{1}^{\prime}\right)$.

Conversely, each $\sigma \in \operatorname{Aut}(S) \cong \mathfrak{S}_{5}$ mapping $\bar{C}$ to $\overline{C^{\prime}}$ and $A_{1}$ to $A_{1}^{\prime}$ is induced by an isomorphism $\Psi: C \stackrel{\sim}{\longrightarrow} C^{\prime}$ such that $\Psi\left(p_{1}\right)=p_{1}^{\prime}, i=1, \ldots, n$.

If $V \subseteq \mathbb{C}\left[x_{1}, x_{2}, x_{3}\right]_{6}$ is the subspace corresponding to curves having double points at the points of $E:=\left\{E_{1}, E_{2}, E_{3}, E_{4}\right\}$, the map $s$ induces an isomorphism $H^{0}\left(S, \mathcal{O}_{S}(2)\right) \cong V$ as representations of $\mathfrak{S}_{5}$. Consider now the incidence variety

$$
X^{\prime}:=\left\{\left(f, A_{1}\right) \in \mathbb{P}(V) \times \mathbb{P}_{\mathbb{C}}^{2} \mid f\left(A_{1}\right)=0\right\},
$$

the irreducible open subscheme $X \subseteq X^{\prime}$ and the open set $\mathcal{U} \subseteq \mathbb{P}_{\mathbb{C}}^{2} \backslash E$ defined in Lemma 2.5.1 of $[\mathrm{C}-\mathrm{F}]$. Since the general element of $\mathbb{P}(V)$ is an irreducible sextic having nodes at the points of $E$, then the same is true for the curve represented by general points in $X$.

The above description yields a rational map $m_{6,1}: X \rightarrow \mathcal{M}_{6,1}$. Remark 1.1 implies that its fibres are exactly the orbits with respect to the above described action of $\mathfrak{S}_{5}$. The same argument used in Section 2.5 of $[\mathrm{C}-\mathrm{F}]$ implies that $m_{6,1}$ is dominant.

As a consequence, there exists a birational equivalence $\mathcal{M}_{6,1} \approx X / \mathfrak{S}_{5}$ and we will obtain the rationality of $\mathcal{M}_{6,1}$ by proving the rationality of the quotient on the right.

We make the following two assertions.

Claim 1.2. The action of $\mathfrak{S}_{5}$ on $S$ is almost free.

Assuming the above Claim we are now ready to prove the following

Theorem A for $g=6$ and $n=1$. The moduli space $\mathcal{M}_{6,1}$ is rational.

Proof. From now on we will make the obvious identification of $\mathbb{P}_{\mathbb{C}}^{2} \backslash E$ with an open subset of $S$. We first recall (see [SB], Corollary 3 ) that $V$ contains both the trivial and the signature representations of $\mathfrak{S}_{5}$. In particular $\mathbb{P}(V) \times S$ contains a unisecant, whose intersection with $X$ is a unisecant itself, hence there exists a $\mathfrak{S}_{5}$-equivariant birational equivalence $X \approx S \times \mathbb{C}^{14}$. Now, by taking into account Claim 1.2 above and Section 4 of [Do], it follows that the first projection $X \approx S \times \mathbb{C}^{14} \rightarrow S$ induces a vector bundle structure $X / \mathfrak{S}_{5} \approx\left(S \times \mathbb{C}^{14}\right) / \mathfrak{S}_{5} \rightarrow S / \mathfrak{S}_{5}$. Since $S$ is rational then $S / \mathfrak{S}_{5}$ is a unirational surface, thus it is rational by a Theorem of Castelnuovo.

Next we turn to the Claim.

Proof of Claim 1.2. We recall that $S$ contains exactly five pencils of conics. In our plane representation of $S$ they correspond to the four pencils of lines through each one of the 
points $E_{i}$ and to the pencil of conics with base points $E_{0}, E_{1}, E_{2}, E_{3}$. In particular, two distinct conics in the same pencil do not intersect and conics in different pencils intersect exactly at one point.

Choose a conic $D \subseteq S$ in the pencil corresponding to the plane conics through the points of $E$. Consider the closure $F$ of the set $\left(\bigcup_{\sigma \in \mathfrak{S}_{5}} \sigma(D)\right) \backslash D$ and notice that $F \cap D$ is a finite number of points. It follows that if $\sigma \in \mathfrak{S}_{5}$ is in the stabilizer of a point $D \backslash F$ then $\sigma(D)=D$, hence $\sigma$ fixes the pencil $|D|$.

Via $s$, the permutation $\sigma$ corresponds to an element of the subgroup of $\mathfrak{S}_{5}$ generated by the projectivities fixing the set $E$, which is isomorphic to $\mathfrak{S}_{4}$. Such a representation of $\mathfrak{S}_{4}$ is the standard permutation representation, whence it is clear that for a general choice of $p \in D \backslash F$ the stabilizer of $p$ is trivial.

\section{The Rationality of $\mathcal{M}_{6, n}$ FOR $2 \leq n \leq 8$}

Let $\left(C, p_{1}, \ldots, p_{n}\right) \in \mathcal{M}_{6, n}$ be general: then $C$ is general in $\mathcal{M}_{6}$, thus it is neither hyperelliptic nor trigonal. As pointed out in the previous section for each such $C$, the canonical system $|K|$ is very ample and we obtain an embedding $\varphi: C \rightarrow \widetilde{C} \subseteq \mathbb{P}_{\mathbb{C}}^{5}$ of degree 10 .

For every choice of general points $p_{1}, p_{2} \in C$ the linear system $\left|K-2 p_{n-1}-p_{n}\right|$ is a $g_{7}^{2}$. The induced map $\varphi: C \rightarrow \bar{C} \subseteq \mathbb{P}_{\mathbb{C}}^{2}$ coincides with the projection of $\widetilde{C}$ from the plane spanned by the tangent line to $C$ at the point $p_{n-1}$ and $p_{n}$.

Its image $\bar{C}$ is a septic in $\mathbb{P}_{\mathbb{C}}^{2}$ passing through the points $A_{i}:=\varphi\left(p_{i}\right), i=1, \ldots, n$.

Proposition 2.1. Under the above hypotheses, the curve $\bar{C}$ is an integral septic with nine nodes $N_{1}, \ldots, N_{9}$. Moreover there exists a unique smooth cubic $E_{\bar{C}}$ such that

$$
\bar{C} \cdot E_{\bar{C}}=2 \sum_{i=1}^{9} N_{i}+2 A_{n-1}+A_{n} .
$$

Every other integral septic $D$ with nine nodes at $N_{1}, \ldots, N_{9}$ and tangent in $A_{n-1}$ to $E_{\bar{C}}$ cuts out residually on such cubic the point $A_{n}$.

Conversely, each integral septic $D \subseteq \mathbb{P}_{\mathbb{C}}^{2}$ with exactly nine nodes $N_{1}, \ldots, N_{9}$ lying on an smooth cubic $E \subseteq \mathbb{P}_{\mathbb{C}}^{2}$ such that $D \cdot E=2 \sum_{i=1}^{9} N_{i}+2 A_{n-1}+A_{n}$ for two suitable points $A_{n-1}, A_{n} \notin\left\{N_{1}, \ldots, N_{9}\right\}$ is obtained in the above way.

Proof. We start by proving the second part of the statement. Let $\ell \subseteq \mathbb{P}_{\mathbb{C}}^{2}$ be a line. Since the embedding $D \subseteq \mathbb{P}_{\mathbb{C}}^{2}$ is given by the linear system

$$
|\ell \cdot D| \subseteq\left|\ell \cdot D+E \cdot D-2 \sum_{i=1}^{9} N_{i}-2 A_{n-1}-A_{n}\right| \subseteq\left|K-2 A_{n-1}-A_{n}\right|,
$$

it suffices to check that $\operatorname{dim}\left|K-2 A_{n-1}-A_{n}\right|=2$. If not, $\left|2 A_{n-1}-A_{n}\right|$ would be a $g_{3}^{1}$ on $D$, but this is impossible by the following classical argument (see [E-C], Libro Quinto, p. 106). For each $B_{1}+B_{2}+B_{3} \in\left|2 A_{n-1}-A_{n}\right|$, consider the linear system $\Sigma_{B_{1}+B_{2}+B_{3}}$ of quartics through the points $B_{1}, B_{2}, B_{3}, N_{1}, \ldots, N_{9}$ and notice that

$$
\operatorname{dim} \Sigma_{B_{1}+B_{2}+B_{3}} \geq \operatorname{dim}\left|K-B_{1}-B_{2}-B_{3}\right|=\operatorname{dim}\left|K-2 A_{n-1}-A_{n}\right| \geq 3 .
$$


The general element $Q \in \Sigma_{B_{1}+B_{2}+B_{3}}$ is smooth and $\Sigma_{B_{1}+B_{2}+B_{3}}$ cuts out on $Q$ residually to $B_{1}+B_{2}+B_{3}+N_{1}+\ldots+N_{9}$ a $g_{4}^{r}$ with $r \geq 2$, which has to be the canonical $g_{4}^{2}$ on $Q \subseteq \mathbb{P}_{\mathbb{C}}^{2}$ cut out by the lines (since $Q$ is smooth). It follows that $B_{1}, B_{2}, B_{3}, N_{1}, \ldots, N_{9}$ lie on a cubic due to a well-known corollary of Noether's $A \Phi+B \Psi$ Theorem (see [Wa], Theorem 7.7). Thus we should have infinitely many cubics through $N_{1}, \ldots, N_{9}$, one for each choice of $B_{1}+B_{2}+B_{3}$, a contradiction if the points $N_{1}, \ldots, N_{9}$ are general.

Clearly $\bar{C}$ is singular and by the genus formula

$$
\sum_{P \in \bar{C}} m_{P}\left(m_{P}-1\right)=18
$$

$m_{P}$ being the multiplicity of $P$ on the curve $\bar{C}$. Thus $\bar{C}$ carries at most four-fold points as singularities. Since $C$, being general, is not trigonal, then $\bar{C}$ carries at most triple points.

Simple parameter computations show that the families of septics with at least either one triple point or a double point with an infinitely near double point have dimension at most 25 , while the family of septic carrying nine pairwise distinct double points has dimension 26. Since we have proved above that each plane septic with such a last configuration of singularities is the projection from a suitable secant plane of its canonical model, it follows that for a general curve $C$ of genus 6 and for general points $p_{1}, p_{2} \in C$, the singularities of $\bar{C}$ are exactly nine pairwise distinct double points. We denote them by $N_{1}, \ldots, N_{9}$.

Such nine nodes $N_{1}, \ldots, N_{9}$ lie necessarily on at least one cubic. Since through nine general points in the plane there is always a septic having them as nodes (it is obtained as projection of its canonical model again by the first part of the proof), it follows that for a general $C$ such a cubic is uniquely determined: we denote it by $E_{\bar{C}}$.

The curve $E_{\bar{C}}$ is integral. If not, it is the union of a line and a conic. Due to degree reasons, the line and the conic contain at most three and seven nodes respectively, thus there are only two possible distinct configurations. Again a parameter computation shows that both such configurations yield families of dimension at most 24 and we can argue as in the previous cases. In the same way one can deduce that $E_{\bar{C}}$ is smooth.

Next, we check that $A_{n-1}, A_{n} \in E_{\bar{C}}$. By construction, for each general line $\ell \subseteq \mathbb{P}_{\mathbb{C}}^{2}$ the divisor $\ell \cdot \bar{C}+2 A_{n-1}-A_{n}$ is in the canonical system. Since the canonical system is cut out on $\bar{C}$ by the adjoints of degree 4 we have the linear equivalence on $\bar{C}$

$$
\ell \cdot \bar{C}+2 A_{n-1}-A_{n} \sim \ell \cdot \bar{C}+E_{\bar{C}} \cdot \bar{C}-2 \sum_{i=1}^{9} N_{i}
$$

Thus $2 A_{n-1}-A_{n} \sim E_{\bar{C}} \cdot \bar{C}-2 \sum_{i=1}^{9} N_{i}$ and since the general curve $\bar{C}$ is not trigonal we have $E_{\bar{C}} \cdot \bar{C}=2 A_{n-1}-A_{n}+2 \sum_{i=1}^{9} N_{i}$, i.e. $A_{n-1}, A_{n} \in E_{\bar{C}}$ and the curves $E_{\bar{C}}$ and $\bar{C}$ are tangent at $A_{n-1}$.

Finally, consider the linear system $\mathcal{L}$ of plane septic curves passing doubly through $N_{1}, \ldots, N_{9}$ and and tangent to $E_{\bar{C}}$ at the point $A_{n-1}$ : by Bezout's theorem, every element of $\mathcal{L}$ intersects the cubic $E_{\bar{C}}$ in another point $A_{n}$. This point has to be fixed, otherwise $\mathcal{L}$ would cut a $g_{1}^{1}$ on $E_{\bar{C}}$. 
Let $V_{7,9} \subseteq\left|\mathcal{O}_{\mathbb{P}_{\mathbb{C}}^{2}}(7)\right|$ be the Severi variety of plane curves of degree 7 with exactly 9 nodes as singularities. We consider the closure $V_{7,9}^{\text {tang }} \subseteq V_{7,9}$ of the locus of those curves which are tangent to the cubic through their nodes at some point.

Proposition 2.1 shows that for each general $D \in V_{7,9}^{\operatorname{tang}}$ then $D=\bar{C}$, thus we obtain two points $A_{n-1}, A_{n} \in D \subseteq \mathbb{P}_{\mathbb{C}}^{2}$ where $A_{n-1}$ is the point of tangency of $D$ with the cubic through its nodes, and $A_{n}$ the residual intersection point. Hence we have a rational map

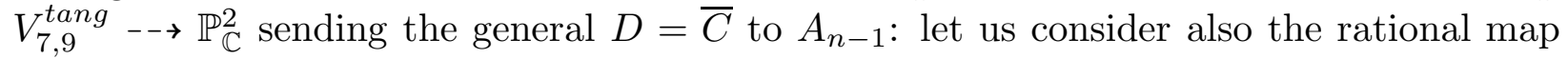
$\left(\mathbb{P}_{\mathbb{C}}^{2}\right)^{n-1} \rightarrow \mathbb{P}_{\mathbb{C}}^{2}$ induced by the projection on the last factor. Thus we may consider the scheme

$$
W_{7,9}^{n}:=\left\{\left(D, A_{1}, \ldots, A_{n-1}\right) \mid A_{1}, \ldots, A_{n-1} \in D\right\} \subseteq V_{7,9}^{\text {tang }} \times_{\mathbb{P}_{\mathbb{C}}^{2}}\left(\mathbb{P}_{\mathbb{C}}^{2}\right)^{n-1}
$$

Now Proposition 2.1 yields the following.

Corollary 2.2. The rational map $\psi: W_{7,9}^{n} \rightarrow \mathcal{M}_{6, n}$ sending $\left(D, A_{1}, \ldots, A_{n-1}\right)$ to its pointed normalization $\left(C, p_{1}, \ldots, p_{n}\right)$ is dominant.

The fibres are the orbits of the natural action of $\mathrm{PGL}_{3}$, hence there is a natural birational equivalence $W_{7,9}^{n} / \mathrm{PGL}_{3} \approx \mathcal{M}_{6, n}$.

Proof. It remains to prove the assertion on the fibres. It is obvious that fibre over a point $\left(C, p_{1}, \ldots, p_{n}\right)$ contains the $\mathrm{PGL}_{3}$-orbits of the plane pointed curve $\left(\bar{C}, A_{1}, \ldots, A_{n-1}\right)$.

Conversely, let $\Psi: C \stackrel{\sim}{\longrightarrow} C^{\prime}$ be an isomorphism of abstract $n$-pointed curves of genus 6 mapping the point $p_{i} \in C$ to $p_{i}^{\prime} \in C^{\prime}$. It carries the canonical system $K$ of $C$ onto the one $K^{\prime}$ of $C^{\prime}$. Thus $\Psi$ induces a projectivity of the canonical space mapping the canonical models $\widetilde{C}$ and $\widetilde{C}^{\prime}$ one onto the other: obviously such a projectivity must map the tangent line at $p_{n-1}$ onto the tangent line at $p_{n-1}^{\prime}$, thus $\Psi$ maps the linear system $\left|K-2 p_{n-1}-p_{n}\right|$ to $\left|K^{\prime}-2 p_{n-1}^{\prime}-p_{n}^{\prime}\right|$, hence it induces via the projections $\varphi$ and $\varphi^{\prime}$ a projectivity of $\mathbb{P}_{\mathbb{C}}^{2}$ sending $\bar{C}$ to $\bar{C}^{\prime}$ and $A_{i}:=\varphi\left(p_{i}\right)$ to $A_{i}^{\prime}:=\varphi^{\prime}\left(p_{i}^{\prime}\right)$.

Therefore we can prove the rationality of $\mathcal{M}_{6, n}$ via standard results on quotients. To this purpose we consider the incidence variety

$$
\mathcal{E}:=\left\{\left(E, A_{1}, \ldots, A_{n-1}, N\right) \mid A_{n-1}, N \in E\right\} \subseteq\left|\mathcal{O}_{\mathbb{P}_{\mathbb{C}}^{2}}(3)\right| \times\left(\mathbb{P}_{\mathbb{C}}^{2}\right)^{n}
$$

Denoting by $S^{9} \mathcal{E}$ the symmetric product over $\left\{\left(E, A_{1}, \ldots, A_{n-1}\right) \mid A_{n-1} \in E\right\}$, we notice the existence of a natural chain of rational maps $\beta \circ \alpha: W_{7,9}^{n} \rightarrow S^{9} \mathcal{E} \rightarrow \mathcal{E}$, where

$$
\alpha\left(D, A_{1}, \ldots, A_{n-1}\right)=\left(E, A_{1}, \ldots, A_{n-1}, N_{1}+\ldots+N_{9}\right)
$$

$N_{1}, \ldots, N_{9}$ being the nodes of $D \in V_{7,9}^{\operatorname{tang}}$ on the smooth cubic $E$,

$$
\beta\left(E, A_{1}, \ldots, A_{n-1}, N_{1}+\ldots+N_{9}\right)=\left(E, A_{1}, \ldots, A_{n-1}, N\right)
$$

the point $N$ being the unique element in $\left|N_{1}+\ldots+N_{9}-8 A_{1}\right|$ on the smooth cubic $E$. 
Lemma 2.3. The action of $\mathrm{PGL}_{3}$ on $\mathcal{E}$ has trivial stabilizer at the general point and the quotient $\mathcal{E} / \mathrm{PGL}_{3}$ is rational.

Proof. Let $\left(E, A_{1}, \ldots, A_{n-1}, N\right) \in \mathcal{E}$ be general. Each projectivity leaving fixed both the curve $E$ and the points $A_{1}, \ldots, A_{n-1}, N$ induces an automorphism of the abstract elliptic curve $E$ fixing $A_{n-1}, N$. Since the automorphism group of the 1-pointed general elliptic curve $(E, N)$ is isomorphic to $\mathbb{Z}_{2}$ (see e.g. [Ha], Corollary IV.4.7), then a general choice of $A_{n-1}$ implies that each element in the stabilizer of $\left(E, A_{1}, \ldots, A_{n-1}, N\right)$ restricts to the identity on $E$, hence has to be the identity on the whole plane since the embedded curve $E \subseteq \mathbb{P}_{\mathbb{C}}^{2}$ contains 4 points in general position.

Let $T \subseteq \mathrm{PGL}_{3}$ be the stabilizer of the points $[1,0,0],[0,0,1]$ and the line $r:=\left\{x_{2}=0\right\}$ in $\mathrm{PGL}_{3}: T$ is a triangular subgroup of $\mathrm{PGL}_{3}$. Clearly

$$
\mathcal{E}_{0}:=\left\{\left(E, A_{1}, \ldots, A_{n-2},[1,0,0],[0,0,1]\right) \in \mathcal{E} \mid E \text { is tangent to } r \text { at }[1,0,0]\right\}
$$

is a $\left(\mathrm{PGL}_{3}, T\right)$-section of $\mathcal{E}$ in the sense of [Ka] (see also Section 3 of [Do] where it is called "slice"). In particular $\mathcal{E} / \mathrm{PGL}_{3} \approx \mathcal{E}_{0} / T$ by [Ka], Proposition 1.2.

Define the vector space $V:=\left\langle x_{0}^{2} x_{2}, x_{0} x_{1}^{2}, x_{0} x_{1} x_{2}, x_{0} x_{2}^{2}, x_{1}^{2} x_{2}, x_{1} x_{2}^{2}, x_{2}^{3}\right\rangle:$ then

$$
\mathcal{E}_{0} \cong \mathbb{P}(V) \times\left(\mathbb{P}_{\mathbb{C}}^{2}\right)^{n-2} \cong\left(V \oplus \mathbb{C}^{\oplus 3(n-2)}\right) /\left(\mathbb{C}^{*}\right)^{n-1}
$$

thus

$$
\mathcal{E} / \mathrm{PGL}_{3} \approx \mathcal{E}_{0} / T \approx\left(\mathbb{P}(V) \times\left(\mathbb{P}_{\mathbb{C}}^{2}\right)^{n-2}\right) / T \approx\left(V \oplus \mathbb{C}^{\oplus 3(n-2)}\right) /\left(\mathbb{C}^{*}\right)^{n-1} \rtimes T
$$

Since the action of $\left(\mathbb{C}^{*}\right)^{n-1} \rtimes T$ on $\left(V \oplus \mathbb{C}^{\oplus 3}\right)$ is linear and triangular, then $\mathcal{E} / \mathrm{PGL}_{3}$ turns out to be rational by $[\mathrm{Vi}]$.

We are now ready to prove the following

Theorem $\mathbf{A}$ for $g=6$ and $2 \leq n \leq 8$. The moduli space $\mathcal{M}_{6, n}$ is rational for $2 \leq n \leq 8$.

Proof. Due to the above construction it suffices to prove that $W_{7,9}^{n} / \mathrm{PGL}_{3}$ is rational if $2 \leq n \leq 8$. The maps $\alpha, \beta$ are trivially $\mathrm{PGL}_{3}$-equivariant by construction. Moreover the action of $\mathrm{PGL}_{3}$ on $S^{9} \mathcal{E}$ has trivial stabilizer at the general point since, otherwise, by projecting down over $\mathcal{E}$ we would obtain points with non-trivial stabilizer.

The typical fibre of $\beta$ over $\left(E, A_{1}, A_{2}, \ldots, A_{n-1}, N\right)$ is given by all divisors $N_{1}+\ldots+N_{9}$ such that $N_{1}+\ldots+N_{9} \in\left|N+8 A_{1}\right|$. In particular $\beta$ is dominant and it is a projective bundle over $\mathcal{E}$ with typical fibre $\mathbb{P}_{\mathbb{C}}^{8}$. The section $\left\{\left(E, A_{1}, A_{2}, \ldots, A_{n}, N+8 A_{1}\right)\right\} \subset S^{9} \mathcal{E}$ is $\mathrm{PGL}_{3}$-equivariant: since $\mathrm{PGL}_{3}$ is reductive there exists a $\mathrm{PGL}_{3}$-invariant hyperplane in each fibre, so Lemma 2.3 above and the "no-name method" (see [Do], Section 4) yield the rationality of the quotient $S^{9} \mathcal{E} / \mathrm{PGL}_{3}$.

The typical fibre of $\alpha$ over $\left(E, A_{1}, A_{2}, \ldots, A_{n-1}, N_{1}+\ldots+N_{9}\right)$ is given by all integral septic curves passing doubly through $N_{1}, \ldots, N_{9}$, simply through $A_{1}, \ldots, A_{n-2}$ and tangent to the conic $E$ at the point $A_{n-1}$ which is a linear system of dimension $8-n$, while the sublocus of non-integral septics has dimension at most $7-n$ : it then follows from Bertini Theorem that the general fibre is non-empty and it is isomorphic to $\mathbb{P}_{\mathbb{C}}^{8-n}$. Let $r$ be the 
tangent line at $E$ in the point $A_{n-1}$. Then the section $\left\{\left(2 E+r, A_{1}, \ldots, A_{n-1}\right)\right\} \subset W_{7,9}^{n}$ is $\mathrm{PGL}_{3}$-equivariant. Thus the same argument as above yields the stated rationality.

Comments to Section 2. If we try to repeat verbatim the above construction for the case $g=7$, we encounter two problems. On one hand, the general fibre of $\alpha$ should be given by all plane octic curves passing doubly through 14 general points and simply through 4 general points, hence it is empty. On the other hand, the morphism $\beta$ cannot be defined exactly in the same way since on a smooth quartic curve there is no group law.

\section{The Unirationality of $\mathcal{M}_{8, n}$ FOR $1 \leq n \leq 11$}

Let $\left(C, p_{1}, \ldots, p_{11}\right) \in \mathcal{M}_{8,11}$ be general. Due to the Dimension Theorem (see $[\mathrm{G}-\mathrm{H}]$ : see also $[\mathrm{A}-\mathrm{C}-\mathrm{G}-\mathrm{H}],(1.5))$, the minimum $d$ for which $C$ carries a $g_{d}^{1}$ is 5 . Moreover it carries exactly 21 distinct $g_{5}^{1}$ by a formula of Castelnuovo (see [Ca]: see also [Fu], Example 14.4.5 or Chapter VII of $[\mathrm{A}-\mathrm{C}-\mathrm{G}-\mathrm{H}])$. By choosing two morphisms $\varphi_{i}: C \rightarrow \mathbb{P}_{\mathbb{C}}^{1}, i=1,2$, corresponding to two fixed $g_{5}^{1}$ 's on $C$, we then obtain a morphism $\varphi:=\left(\varphi_{1}, \varphi_{2}\right): C \rightarrow Q:=$ $\mathbb{P}_{\mathbb{C}}^{1} \times \mathbb{P}_{\mathbb{C}}^{1}$

We first notice that $\varphi$ has either degree 5 or it is birational onto its image. The first case occurs if and only if $\varphi_{1}$ and $\varphi_{2}$ are induced by the same $g_{5}^{1}$. Thus we can assume that $\varphi$ is birational onto its image from now on. By construction $\varphi(C) \in\left|\mathcal{O}_{Q}(5,5)\right|$ and it is naturally endowed with an ordered 11-tuple $\left(A_{1}, \ldots, A_{11}\right)$ of points, namely $A_{i}:=\varphi\left(p_{i}\right)$. Thus the arithmetic genus of $\varphi(C)$ is 16 , and the genus formula then yields

$$
\sum_{N \in \varphi(C)} \frac{m_{N}\left(m_{N}-1\right)}{2}=16-8=8
$$

where $m_{N}$ denotes the multiplicity of the point $N \in \varphi(C)$. It follows that $\varphi(C)$ is necessarily singular and it carries at most fourfold points. The linear system of conics through all the points of multiplicity greater than two would cut on $\varphi(C)$ linear series with negative Brill-Noether number. Since $C$ is general this is not possible (again by the Dimension Theorem), thus $\varphi(C)$ carries at most double points as singularities. Let us consider the incidence variety

$$
\begin{gathered}
\mathcal{I}:=\left\{\left(D, N_{1}, \ldots, N_{8}, A_{1}, \ldots, A_{11}\right) \in\left|\mathcal{O}_{Q}(5,5)\right| \times Q^{19} \mid\right. \\
\left.A_{i} \in D, N_{j} \text { is double on } D\right\} \stackrel{\alpha}{\longrightarrow} Q^{19}
\end{gathered}
$$

(in this notation a point $N_{j}$ is repeated $t$ times if it is double and there are $t-1$ other double points infinitely near to it).

Proposition 3.1. For the general point of $\left(D, N_{1}, \ldots, N_{8}, A_{1}, \ldots, A_{11}\right) \in \mathcal{I}$, the curve $D$ is irreducible with no singularities but nodes at $N_{1}, \ldots, N_{8}$, hence its normalization is a 11-pointed curve of genus 8.

Proof. We have only to check the more or less obvious fact that for the general point $\left(D, N_{1}, \ldots, N_{8}, A_{1}, \ldots, A_{11}\right) \in \mathcal{I}$ the curve $D$ is irreducible and it has no singularities but the nodes $N_{1}, \ldots, N_{8}$. 
Chose a general 8-tuple $\left(N_{1}, \ldots, N_{8}\right) \in Q^{8}$ of pairwise distinct points. Then there exists a smooth irreducible element $D^{\prime} \in\left|\mathcal{O}_{Q}(2,3)\right|$ through $N_{1}, \ldots, N_{8}$. For each other point $A \in Q$ there exists a divisor $D^{\prime \prime} \in\left|\mathcal{O}_{Q}(3,2)\right|$ such that $D^{\prime}+D^{\prime \prime} \in\left|\mathcal{O}_{Q}(5,5)\right|$ is smooth at $A$. Thus the general $D$ has no singularities but $N_{1}, \ldots, N_{8}$.

Due to the exact sequence

$$
0 \longrightarrow \mathcal{O}_{Q}(1,-1) \longrightarrow \mathcal{O}_{Q}(3,2) \longrightarrow \mathcal{O}_{Q}(1,-1) \otimes \mathcal{O}_{D^{\prime}} \longrightarrow 0
$$

one infers that the very ample linear system $\left|\mathcal{O}_{Q}(1,-1) \otimes \mathcal{O}_{D^{\prime}}\right|$ is cut out by $\left|\mathcal{O}_{Q}(1,-1)\right|$. Since the general divisor in $\left|\mathcal{O}_{Q}(1,-1) \otimes \mathcal{O}_{D^{\prime}}\right|$ has only simple points in its support then we are able to find an element $D^{\prime \prime} \in\left|\mathcal{O}_{Q}(3,2)\right|$ intersecting transversaly $D^{\prime}$ at $N_{1}, \ldots, N_{8}$, whence $D^{\prime}+D^{\prime \prime} \in\left|\mathcal{O}_{Q}(5,5)\right|$ has nodes at the points $N_{1}, \ldots, N_{8}$. Thus the general $D$ has nodes at the points $N_{1}, \ldots, N_{8}$.

Now an easy computation shows that a curve in $\left|\mathcal{O}_{Q}(5,5)\right|$ with exactly eight nodes as singularities cannot be reducible.

From the construction above and Proposition 3.1 it follows that

Corollary 3.2. The composition of the forgetful map onto $\left|\mathcal{O}_{Q}(5,5)\right| \times Q^{19}$ with the normalization gives a dominant rational map $m: \mathcal{I}_{n} \rightarrow-\rightarrow \mathcal{M}_{19}$.

The fibre of $\alpha$ over a point in $Q^{19}$ is a possibly empty subspace of $\left|\mathcal{O}_{Q}(5,5)\right|$. Since each double point imposes three linear conditions on the divisors through it, then there exists an open set $\mathcal{V} \subseteq Q^{19}$ non-intersecting the diagonals of $Q^{19}$ over which the fibres of $\alpha$ are projective spaces of constant dimension $\operatorname{dim}\left|\mathcal{O}_{Q}(5,5)\right|-24-11=0$, i.e. a single point: in particular $\mathcal{I}$ contains an irreducible component

$$
\mathcal{I}^{\text {gen }} \subseteq\left|\mathcal{O}_{Q}(5,5)\right| \times Q^{19}
$$

of dimension 38 which is birational via $\alpha$ to a non-empty open subset $\mathcal{U} \subseteq \mathcal{V} \subseteq Q^{19}$, thus it is irreducible and rational.

The fibres of $\alpha$ containing curves of $\left|\mathcal{O}_{Q}(5,5)\right|$ with infinitely near points map over some diagonal in $Q^{19}$. Since a double point infinitely near to a double point imposes five linear conditions on the divisors through it then the union of such fibres is a subscheme $\mathcal{I}^{s p} \subseteq \mathcal{I}$ of dimension at most 37 .

Since on $\mathcal{I}$ there is a natural action of the group of automorphisms $\operatorname{Aut}(Q)$, which has dimension 6 , then the fibres of $m$ have at least dimension 6 , thus $\mathcal{I}^{s p}$ maps onto a proper subscheme of $\mathcal{M}_{8,11}$. It follows

Theorem B for $g=8$. The moduli space $\mathcal{M}_{8, n}$ is unirational for $1 \leq n \leq 11$.

Proof. The restriction of $m$ to $\mathcal{I}^{g e n}$ is dominant. Since $\mathcal{I}^{\text {gen }}$ is rational and dominates $\mathcal{M}_{8,11}$, then $\mathcal{M}_{8,11}$ turns out to be unirational. Since the forgetful map $\mathcal{M}_{8,11} \rightarrow \mathcal{M}_{8, n}$ is dominant for $n \leq 10$, it turns out the unirationality of $\mathcal{M}_{8, n}$ for $1 \leq n \leq 10$ too.

\section{The Unirationality of $\mathcal{M}_{10, n}$ FOR $1 \leq n \leq 3$}

Let $\left(C, p_{1}, p_{2}, p_{3}\right) \in \mathcal{M}_{10,3}$ be general: $C$ carries exactly 42 distinct $g_{6}^{1}$ by the formula of Castelnuovo quoted above. In $[\mathrm{Se}]$ (see also $[\mathrm{A}-\mathrm{C}]$ ) it is proved the existence of a morphism 
$\varphi: C \rightarrow \mathbb{P}_{\mathbb{C}}^{2}$ whose image $\varphi(C) \subseteq \mathbb{P}_{\mathbb{C}}^{2}$ is a curve of degree 9 with one ordinary triple point and 15 nodes and no other singularities. Let us consider the incidence variety

$$
\begin{aligned}
\mathcal{I}:=\left\{\left(D, N_{0}, N_{1} \ldots, N_{15}, A_{1}, A_{2}, A_{3}\right) \in\left|\mathcal{O}_{\mathbb{P}_{\mathbb{C}}^{2}}(9)\right| \times\left(\mathbb{P}_{\mathbb{C}}^{2}\right)^{19} \mid\right. \\
\left.\quad A_{i} \in D, N_{0} \text { is triple on } D, N_{j} \text { is double on } D \text { for } j>0\right\} \stackrel{\alpha}{\longrightarrow}\left(\mathbb{P}_{\mathbb{C}}^{2}\right)^{19}
\end{aligned}
$$

(in this notation a point $N_{j}$ is repeated $t$ times if it is double and there are $t-1$ other double points infinitely near to it). Corollary 4.7 of $[\mathrm{A}-\mathrm{C}]$ in the particular case $n=9$, $d=6$ and $\delta=15$ yields the following

Proposition 4.1. For the general point $\left(D, N_{0}, N_{1} \ldots, N_{15}, A_{1}, A_{2}, A_{3}\right) \in \mathcal{I}$, the curve $D$ is irreducible with no singularities but an ordinary triple point at $N_{0}$ and nodes at $N_{1}, \ldots, N_{15}$, hence its normalization is a $n$-pointed curve of genus 10 .

From the construction above and Proposition 4.1 it follows that

Corollary 4.2. The composition of the forgetful map onto $\left|\mathcal{O}_{\mathbb{P}_{\mathbb{C}}^{2}}(9)\right| \times\left(\mathbb{P}_{\mathbb{C}}^{2}\right)^{19}$ with the normalization gives a dominant rational map $m: \mathcal{I} \rightarrow \rightarrow \mathcal{M}_{10,3}$.

As in the genus 8 case, then $\mathcal{I}$ contains an irreducible component

$$
\mathcal{I}^{\text {gen }} \subseteq\left|\mathcal{O}_{\mathbb{P}_{\mathbb{C}}^{2}}(9)\right| \times\left(\mathbb{P}_{\mathbb{C}}^{2}\right)^{19}
$$

of dimension 38 which is birational to a non-empty open subset $\mathcal{U} \subseteq\left(\mathbb{P}_{\mathbb{C}}^{2}\right)^{19}$ not intersecting the diagonals of $\left(\mathbb{P}_{\mathbb{C}}^{2}\right)^{19}$, thus it is irreducible and rational. Again the locus of points in $\mathcal{I}$ carrying infinitely near singularities has dimension at most 37 , thus

Theorem B for $g=10$. The moduli space $\mathcal{M}_{10, n}$ is unirational for $1 \leq n \leq 3$.

Proof. The restriction of $m$ to $\mathcal{I}^{\text {gen }}$ is dominant. Since $\mathcal{I}^{\text {gen }}$ is a projective bundle dominating $\mathcal{M}_{10,3}$, then $\mathcal{M}_{10,3}$ turns out to be unirational. Since the forgetful map $\mathcal{M}_{10,3} \rightarrow \mathcal{M}_{10, n}$ is dominant for $n \leq 2$, it turns out the unirationality of $\mathcal{M}_{10, n}$ for $1 \leq n \leq 2$ too.

Comments to Sections 3 and 4. Our proof of the unirationality of $\mathcal{M}_{g, n}$ depends on the existence of models with a particular configuration of singularities. In the two cases $g=$ 8, 10 we used two different models and it is natural to ask why we made such a choice.

For instance we could embed the general curve of genus $g=8$ in $\mathbb{P}_{\mathbb{C}}^{2}$ as a plane curve of degree 8 with a triple point and 10 nodes and prove the unirationality of $\mathcal{M}_{8, n}$ as for $g=10$. However, a standard easy parameter count shows that this approach yields the unirationality up to $n=8$ which is in any case greater than Logan's bound but lower than the value obtained with the model on a quadric.

Projecting our model from a double point onto a plane we obtain again a curve of degree 8 with 2 triple points and only 7 double points. Since a triple point imposes on curves of given degree less conditions than 3 double points, we are able with this model to improve the unirationality result.

With this remark in mind one could hope to construct plane models with more triple points. Unfortunately an easy parameter count shows that the locus in $\mathcal{M}_{8}$ of curves that can be represented as plane curve of degree 8 with at least 3 triple points has codimension 1 , so our result is the best possible via our method. 
When $g=10$ we can also try to argue as in the case $g=8$ by mapping birationally a general curve $C$ of genus 10 onto a singular curve of bidegree $(6,6)$ on a smooth quadric. In this case one expects 15 nodes as singularities but this cannot be proved with the same method we used for $g=8$. Indeed, in this case the Brill-Noether number can be non negative even if triple points occur on the image of $C$. On the other hand, even if we are able to prove that for general $C$ all the singularities are double points and thus even if it makes sense to consider an incidence defined as in the genus 8 case, it is not evident a priori that the general fibre of such morphism contains irreducible curves of genus 10 . Moreover even if all would work in the right way, such an approach would yield the same bound $n \leq 3$ !

Thus it is natural to ask if it is possible to represent curves of genus 10 as plane curves of degree 9 with at least two triple points. Again an easy parameter count shows that the locus of such curves in $\mathcal{M}_{10}$ has codimension 1.

\section{The Unirationality of $\mathcal{M}_{12,1}\left(\right.$ And of $\mathcal{M}_{14, n}$ FOR $\left.1 \leq n \leq 2\right)$}

We first recall the main steps of Verra's proof of the unirationality of $\mathcal{M}_{g}$ for $g=12,14$ (see $[\mathrm{Ve}]$ ), also in order to fix the notation used throughout this section.

Let $\mathcal{H} i l b_{p(t)}\left(\mathbb{P}_{\mathbb{C}}^{r}\right)$ be the Hilbert scheme parameterizing subschemes $X \subseteq \mathbb{P}_{\mathbb{C}}^{r}$ with Hilbert polynomial $p(t)$.

Definition 5.1. For each triple of positive integers $(d, g, r)$, we denote by $\mathcal{C}_{d, g, r}$ the subset of of all smooth curves $C \in \mathcal{H} i l b_{d t+1-g}\left(\mathbb{P}_{\mathbb{C}}^{r}\right)$ such that $\mathcal{O}_{C}(1)$ is non-special and the rank of the natural restriction morphisms $H^{0}\left(\mathbb{P}_{\mathbb{C}}^{r}, \mathcal{O}_{\mathbb{P}_{\mathbb{C}}^{r}}(f)\right) \rightarrow H^{0}\left(C, \mathcal{O}_{C}(f)\right)$ is maximal for each $f \in \mathbb{Z}$.

Clearly $\mathcal{C}_{d, g, r} \subseteq \mathcal{H} i l b_{d t+1-g}\left(\mathbb{P}_{\mathbb{C}}^{r}\right)$ is open and it is non-empty if $3 \leq r \leq d-g$ due to [B-E1], [B-E2], [B-E3]. Moreover, in Section 1 of [Ve], it is also proved the following

Lemma 5.2. If $g \leq 9$ then $\mathcal{C}_{d, g, r}$ is irreducible and unirational.

Due to the definition of $H^{0}\left(\mathbb{P}_{\mathbb{C}}^{r}, \Im_{C}(f)\right)$, for each $f \in \mathbb{Z}$ the dimension $h^{0}\left(\mathbb{P}_{\mathbb{C}}^{r}, \Im_{C}(f)\right)$ is constant for $C \in \mathcal{C}_{d, g, r}$, thus there exists a positive integer $f$ satisfying $h^{0}\left(\mathbb{P}_{\mathbb{C}}^{r}, \Im_{C}(f)\right) \geq$ $r-1$ for each $C \in \mathcal{C}_{d, g, r}$ : from now on we will assume that the choice of such an $f$ has been made.

Let $\mathcal{G}_{d, g, r} \rightarrow \mathcal{C}_{d, g, r}$ be the Grassmann bundle parameterizing pairs $(C, V)$ such that $C \in \mathcal{C}_{d, g, r}$ and $V \subseteq H^{0}\left(\mathbb{P}_{\mathbb{C}}^{r}, \Im_{C}(f)\right)$ has dimension $r-1$. For each point $(C, V) \in \mathcal{G}_{d, g, r}$ let $B_{(C, V)}$ be the base locus of the linear system of hypersurfaces associated to $V$.

Definition 5.3. Let $n_{f, d, g, r}:=h^{0}\left(\mathbb{P}_{\mathbb{C}}^{r}, \Im_{C}(f)\right)-(r-1)$ and define the incidence variety

$$
\begin{aligned}
\mathcal{I}_{f, d, g, r}:=\{ & \left(C, V, A_{1}, \ldots, A_{n}\right) \in \mathcal{G}_{d, g, r} \times\left(\mathbb{P}_{\mathbb{C}}^{r}\right)^{n_{f, d, g, r}} \mid \\
& \left.A_{i} \in B_{(C, V)}, i=1, \ldots, n_{f, d, g, r}\right\} \stackrel{\alpha}{\longrightarrow} \mathcal{C}_{d, g, r} \times\left(\mathbb{P}_{\mathbb{C}}^{r}\right)^{n_{f, d, g, r}} .
\end{aligned}
$$

Points in $\mathcal{I}_{f, d, g, r}$ are $\left(n_{f, d, g, r}+2\right)$-tuples $\left(C, V, A_{1}, \ldots, A_{n_{f, d, g, r}}\right)$ such that $C \in \mathcal{C}_{d, g, r}$ and $V \subseteq H^{0}\left(\mathbb{P}_{\mathbb{C}}^{r}, \Im_{C}(f)\right)$ has dimension $r-1$. By definition, $C \subseteq B_{(C, V)}$ and $B_{(C, V)}$ could be a curve. If this is the case then $B_{(C, V)}$ is a complete intersection curve and, due to 
the definition of $n_{f, d, g, r}, V$ is exactly the subspace of $H^{0}\left(\mathbb{P}_{\mathbb{C}}^{r}, \Im_{C}(f)\right)$ corresponding to hypersurfaces through $C$ and $A_{1}, \ldots, A_{n_{f, d, g, r}}$. In particular, in this case $\alpha$ is generically injective, thus it is birational since it is surjective by construction.

Moreover, there is also an algebraic linkage $B_{(C, V)}=C \cup D_{(C, V)}$, where $D_{(C, V)}$ is also a curve. If $B_{(C, V)}$ is nodal and $D_{(C, V)}$ is smooth, irreducible and non-degenerate in $\mathbb{P}_{\mathbb{C}}^{r}$ then its degree $d^{\prime}$ and genus $g^{\prime}$ are given by the formulas $d+d^{\prime}=f^{r-1}$ and $2\left(g-g^{\prime}\right)=$ $(f(r-1)-r-1)\left(d-d^{\prime}\right)($ see $[\mathrm{Mi}]$, Corollaries 5.2.13 and 5.2.14).

Let us restrict ourselves to the case $(f, d, g, r)=(2,15,9,6)$. Notice that in this case $h^{0}\left(\mathbb{P}_{\mathbb{C}}^{6}, \mathcal{O}_{\mathbb{P}_{\mathbb{C}}^{6}}(2)\right)=28>22=h^{0}\left(C, \mathcal{O}_{C}(2)\right)$ : since $C$ has maximal rank then

$$
n_{2,15,9,6}:=h^{0}\left(\mathbb{P}_{\mathbb{C}}^{6}, \Im_{C}(2)\right)-5=h^{0}\left(\mathbb{P}_{\mathbb{C}}^{6}, \mathcal{O}_{\mathbb{P}_{\mathbb{C}}^{6}}(2)\right)-h^{0}\left(C, \mathcal{O}_{C}(2)\right)-5=1
$$

Proposition 5.4. There exists an open subset $\mathcal{U} \subseteq \mathcal{C}_{15,9,6} \times \mathbb{P}_{\mathbb{C}}^{6}$ such that for each $\left(C, V, A_{1}\right) \in \mathcal{I}_{2,15,9,6}^{\text {gen }}:=\alpha^{-1}(\mathcal{U})$, then $D_{(C, V)}$ is smooth, irreducible and non-degenerate in $\mathbb{P}_{\mathbb{C}}^{6}$ with degree 17 and genus 12 .

Proof. There is a dominant morphism $g: \mathcal{I}_{2,15,9,6} \rightarrow \mathcal{G}_{15,9,6}$. In Section 7 of [Ve] it has been proved the existence of an open set $\mathcal{V} \subseteq \mathcal{G}_{15,9,6}$ such that for each $(C, V) \in \mathcal{V}$ the curve $D_{(C, V)}$ satisfies the assertion, hence the same is true for each general point $\left(C, V, A_{1}\right) \in g^{-1}(\mathcal{V})$.

In particular, as in the previous sections, we infer that $\mathcal{I}_{2,15,9,6}^{g e n}$ is birational via $\alpha$ to $\mathcal{U}$ which is unirational due to Lemma 5.2. We are now ready to prove our

Theorem B for $g=12$. The moduli space $\mathcal{M}_{12,1}$ is unirational.

Proof. Thnaks to Proposition 5.4, we have a rational map $m_{2,15,9,6}: \mathcal{I}_{2,15,9,6}^{\text {gen }} \rightarrow \mathcal{M}_{12,1}$, hence it suffices to check it is dominant.

Let $(D, p) \in \mathcal{M}_{12,1}$ be general. Then $D \in \mathcal{M}_{12}$ is general too, thus [Ve], Theorem 4.5 (see Section 7 for its application in this case) implies the existence of $(C, V) \in \mathcal{G}_{15,9,6}$ such that $D_{(C, V)}=D$. Let $A \in D_{(C, V)} \subseteq \mathbb{P}_{\mathbb{C}}^{6}$ be the point corresponding to $p$ via such an isomorphism: since $A \in D_{(C, V)} \subseteq B_{(C, V)}$ and it is general on $D_{(C, V)}$, then $p \notin C, V$ is exactly the space associated to the linear system of hypersurfaces of degree 2 through $C$ and $A$, i.e. $(C, V, A) \in \mathcal{I}_{2,15,9,6}^{g e n}$. By construction $m_{2,15,9,6}(C, V, A)=(D, p)$.

Remark 5.5. In [B-V], Theorem 4.7 the authors prove with the above procedure the unirationality of $\mathcal{M}_{14, n}$ for $1 \leq n \leq 2$. Indeed, due to the result of Section 6 of [Ve], Proposition 5.4 holds true also in the case $(f, d, g, r)=(2,14,8,6)$. In this case $n_{2,14,8,6}=2$ and $D_{(C, V)}$ is a curve of genus 14 , thus one can define the rational map $m_{2,14,8,6}: \mathcal{I}_{2,14,8,6}^{g e n} \rightarrow$ $\mathcal{M}_{14,2}$. As in the case $g=12$ one then checks that $m_{2,14,8,6}$ is dominant.

Comments to Section 5. One could hope to generalize the above construction also for other genera. Reading [Ve] one checks that this is possible if the hypotheses of Theorem 4.5 of $[\mathrm{Ve}]$ are satisfied. This occurs if the sequence of non-negative integers $\left(r, f, d, g, d^{\prime}, g^{\prime}\right)$ 
satisfies the following system

$$
\left\{\begin{array}{l}
f=\frac{r+2}{r-2} \in \mathbb{Z} \\
d+d^{\prime}=f^{r-1} \\
d-g=r-1 \\
2\left(g-g^{\prime}\right)=((r-1) f-r-1)\left(d-d^{\prime}\right) \\
g^{\prime}-(r+1)\left(g^{\prime}-d^{\prime}+r\right) \geq 0
\end{array}\right.
$$

Solving the above system in $\mathbb{Z}$ one then finds all its solutions, which are

$$
\begin{gathered}
(6,2,15,9,17,12), \quad(6,2,14,8,18,14), \quad(6,2,16,10,16,10), \\
(4,3,12,8,15,14), \quad(4,3,13,9,14,11),
\end{gathered}
$$$$
(3,5,12,9,13,12) \text {. }
$$

The first two ones have been used in the present section to prove the unirationality of $\mathcal{M}_{12,1}$ and $\mathcal{M}_{14,2}$. In the third case $n_{2,16,10,6}=0$, we cannot apply the above method with the fourth sequence and the result in Section 4 remains the best possible with the method used in this paper.

Since, we already know (see the table with the values of the functions $\sigma$ and $\tau$ in the introduction) that $\mathcal{M}_{11,10}$ is unirational but $\kappa\left(\mathcal{M}_{11,10}\right) \geq 0$, we cannot improve the known result in genus $g=11$ applying the above method in the fourth case $\left(r, f, d, g, d^{\prime}, g^{\prime}\right)=$ $(4,3,13,9,14,11)$.

The fifth sequence has been used in [Ve] to prove the unirationality of $\mathcal{M}_{13}$ : unfortunately the method of the proof in [Ve] does not allow us to fix points in order to infer the unirationality $\mathcal{M}_{13, n}$ for whatever value of $n$.

Consider finally the last sequence. Since $n_{f, d, g, r}=2$ in this case, the above method would lead to the unirationality of $\mathcal{M}_{12,2}$, improving our result. Therefore, it would be very interesting to understand if at least one of the sufficient conditions of Theorem 4.5 in [Ve] is satisfied.

The first condition is not satisfied since the homogeneous ideal $\oplus_{n \in \mathbb{Z}} H^{0}\left(\mathbb{P}_{\mathbb{C}}^{3}, \Im_{C}(n)\right)$ in $\mathbb{P}_{\mathbb{C}}^{3}$ of a general point $C$ of the family $\mathcal{C}_{12,9,3}$ is minimally generated by sextics other than quintics. Indeed $h^{0}\left(C, \mathcal{O}_{C}(5)\right)=52, h^{0}\left(C, \mathcal{O}_{C}(6)\right)=64, h^{0}\left(\mathbb{P}_{\mathbb{C}}^{3}, \mathcal{O}_{\mathbb{P}_{\mathbb{C}}^{3}}(5)\right)=56$, $h^{0}\left(\mathbb{P}_{\mathbb{C}}^{3}, \mathcal{O}_{\mathbb{P}_{\mathbb{C}}^{3}}(6)\right)=84$, hence the maximal rank condition yields $h^{0}\left(\mathbb{P}_{\mathbb{C}}^{3}, \Im_{C} \mathbb{P}_{\mathbb{C}}^{3}(5)\right)=4$, $h^{0}\left(\mathbb{P}_{\mathbb{C}}^{3}, \Im_{C}(6)\right)=20$.

Thus the only chance is to verify the second condition. A careful reading of Verra's construction will convince the interested reader that instead of checking the irreducibility of the universal Brill-Noether locus $\mathcal{W}_{13,12}^{3}$, it suffices to use the irreducibility of the subscheme $\mathcal{J}^{\prime}(13,12,3)$ of the Hilbert scheme of curves in $\mathbb{P}_{\mathbb{C}}^{3}$, whose general point corresponds to a smooth, irreducible and non-degenerate curve of degree 13 and genus 12 (see [K-K], Theorem 2.7 (iii)).

Hence the crucial point it seems to establish if $\mathcal{G}_{12,9,3}$ satisfies the key condition. In principle one could try to apply [Ve], Proposition 3.4, but the assumption that the twisted sheaf $\Im_{C}(5)$ of ideals of a general point $C$ in $\mathcal{C}_{12,9,3}$ is globally generated is hard to be 
verified. Another possibility could be to address directly the key condition but again this seems to be completely out of reach for us. Therefore we leave it as an intriguing open problem.

\section{REFERENCES}

[A-C] E. Arbarello, M. Cornalba, Footnotes to a paper of Beniamino Segre, Math. Ann. 256 (1981), 341-362.

[A-C-G-H] E. Arbarello, M. Cornalba, P.A. Griffiths, J. Harris, Geometry of algebraic curves, vol. I, Springer, 1985.

[B-E1] E. Ballico, Ph. Ellia, On postulation of curves in $\mathbb{P}^{4}$, Math. Z. 188 (1985), 355-367.

[B-E2] E. Ballico, Ph. Ellia, The maximal rank conjecture for non-special curves in $\mathbb{P}^{3}$, Invent. Math. 79 (1985), 541-555.

[B-E3] E. Ballico, Ph. Ellia, The maximal rank conjecture for non-special curves in $\mathbb{P}^{n}$, Math. Z. 196 (1987), 355-367.

[Be] P. Belorousski, Chow rings of moduli spaces of pointed elliptic curves, P.H.D. thesis, Chicago, 1998.

[B-F] G. Bini, C. Fontanari, Moduli of curves and spin structures via algebraic geometry, Trans. Amer. Math. Soc. 358 (2006), 3207-3217.

[B-V] A. Bruno, A. Verra, $\mathcal{M}_{15}$ is rationally connected, Projective varieties with unexpected properties (2005), 51-65.

[C-F] G. Casnati, C. Fontanari, On the rationality of moduli spaces of pointed curves, J. London Math. Soc. (to appear).

[Ca] G. Castelnuovo, Numero delle involuzioni razionali giacenti sopra una curva di dato genere, Rend. R. Accad. Lincei V (1889), 130-133.

[Do] I.V. Dolgachev, Rationality of the fields of invariants, Algebraic Geometry, Bowdoin 1985 (Spencer J. Bloch, ed.), Proceedings of Symposia in Pure Mathematics, vol. 46, A.M.S., 1987, pp. $3-16$.

[E-C] F. Enriques, O. Chisini, Lezioni sulla teoria geometrica delle equazioni e delle funzioni algebriche, Zanichelli, 1924.

[Fa] G. Farkas, Koszul divisors on moduli spaces of curves, Pre-primt math.AG/0607475.

[Fu] W. Fulton, Intersection theory, Ergebnisse der Mathematik und ihrer Grenzgebiete (3), vol. 2., Springer, 1984.

[G-H] P.A. Griffiths, J. Harris, The dimension of the variety of special linear systems on a general curve, Duke Math. J. 47 (1980), 233-272.

[Ha] R. Hartshorne, Algebraic geometry, GTM 52, Springer, 1977.

[Ka] P.I. Katsylo, The rationality of the orbit spaces of irreducible representations of the group $S L_{2}$, Math. USSR Izvestiya 22 (1984), 23-32.

[K-K] C. Keem, S. Kim, Irreducibility of a subscheme of the Hilbert scheme of complex space curves, J. Algebra 145 (1992), 240-248.

[Lg] A. Logan, The Kodaira dimension of moduli spaces of curves with marked points, Am. J. Math. 125 (2003), 105-138.

[Mi] J.C. Migliore, Introduction to liaison theory and deficiency modules, Progress in Mathematics 165, Birkhäuserr, 1998.

[Se] B. Segre, Sui moduli delle curve poligonali e sopra un complemento al teorema di esistenza di Riemann, Math. Ann. 100 (1928), 537-551.

[SB] N.I. Shepherd-Barron, Invariant theory for $S_{5}$ and the rationality of $M_{6}$, Compositio Math. 70 (1989), 13-25.

[Ve] A. Verra, The unirationality of the moduli spaces of curves of genus 14 or lower, Compos. Math. 141 (2005), 1425-1444.

[Vi] E.B. Vinberg, Rationality of the field of invariants of a triangular group, Mosc. Univ. Math. Bull. 37 (1982), 27-29. 
[Wa] R. Walker, Algebraic curves, Oxford U.P., 1950.

Edoardo Ballico, Dipartimento di Matematica, Università degli Studi di Trento, Via Sommarive 14, 38050 Povo, Italy

E-mail address: ballico@science.unitn.it

Gianfranco Casnati, Dipartimento di Matematica, Politecnico di Torino, C.so Duca degli Abruzzi 24, 10129 Torino, ItAly

E-mail address: casnati@calvino.polito.it

C. Fontanari, Dipartimento di Matematica, Politecnico di Torino, c.so Duca Degli AbruzZI 24, 10129 TORINO, ITALY

E-mail address: claudio.fontanari@polito.it 